\title{
A Monochromatic electron neutrino beam
}

\author{
Mats Lindroos* \\ CERN, Switzerland \\ E-mail: mats.lindroos@cern.ch
}

\section{J. Bernabeu}

Valencia University, Valencia, Spain

E-mail: jose.bernabeu@uv.es

\section{J. Burguet-Castell}

Valencia University, Valencia, Spain

E-mail: burguet@cern.ch

\section{Espinoza}

Valencia University, Valencia, Spain

E-mail: esmaca@alumni.uv.es

In the last few years spectacular results have been achieved with the demonstration of non vanishing neutrino masses and flavour mixing. Here, a novel method to create a monochromatic neutrino beam, an old dream for neutrino physics, is described based on the recent discovery of nuclei with fast decay through electron-capture to Gamow-Teller resonances in super allowed transitions. Such nuclei will generate a monochromatic directional neutrino beam when decaying at high energy in a storage ring with long straight sections. A facility for generating neutrino beams through nuclear decay is under study within the EURISOL Design Study within the $7^{\text {th }}$ European research framework programme. However, the required electron-capture facility will need a different approach to acceleration and storage of the ion beam compared to the standard beta-beam, as the ions cannot be fully stripped. Electron-capture decay requires partly charged ions which will have a short vacuum life-time due to a large cross-section for stripping through collisions with rest gas molecules in the accelerators. First results for production cross-sections, ion cooling and accumulation schemes and stacking schemes will be presented together with a descriptions of the on-going work on the standard beta-beam facility.

International Europhysics Conference on High Energy Physics

July 21st - 27th 2005

Lisboa, Portugal

${ }^{*}$ I acknowledge the financial support of the EC under the FP6 "Research Infrastructure Action- Structuring the European Research Area” EURISOL DS Project Contract no. 515768 RIDS. The EC is not liable for any use that may be made of the information contained herein.

†Speaker. 


\section{Introduction}

The beta-beam concept for the generation of an electron (anti-)neutrino beam was proposed [1] in 2002. A first study [2,3] of the possibility of using the existing CERN machines for the acceleration of radioactive ions to a relativistic gamma of roughly 100, for later storage in a new decay ring of approximately the size of SPS, was made in 2002. The results from this very first short study were very encouraging, but as no resources could be allocated at CERN for this work, it was not continued. In 2004 it was decided to incorporate a design study for the beta-beam within the EURISOL DS proposal. EURISOL [4] is a project name for a next-generation radioactive beam facility based on the ISOL method [5] for the production of intense radioactive beams for nuclear physics, astrophysics and other applications. The proposal was accepted with the beta-beam task as an integral part. The design study officially started 1 February 2005 and will run for 4 years resulting in a conceptual design report for a beta-beam facility as one potential user of EURISOL.

\section{The beta-beam concept and the first study at CERN}

The first study of the feasibility of using the existing CERN accelerator complex for a betabeam facility was made over a few months with very limited manpower (see figure 1). The main objectives were twofold: i) to identify a possible scenario for bunching, acceleration and storage in a few very short bunches of a sufficient amount of radioactive ions for a beta-beam and ii) to identify possible bottlenecks in the proposed scheme. A main objection raised early on concerned the possible activation of the accelerators. Consequently, some time was spent to simulate the activation problem in the decay ring and to calculate the average losses in the accelerator chain. The study proposed to use a thick ISOL target for production of ${ }^{6} \mathrm{He}$ and ${ }^{18} \mathrm{Ne}$ as both isotopes can be produced in large quantities and are easy to handle. Neither of the isotopes have any longlived daughter products that could create a problem in the low-energy part of the facility. Several iterations were required for the "bunching" but eventually a high frequency $(60 \mathrm{GHz})$ ECR source was identified as a possible highly efficient tool to create sufficiently short bunches after the target for multi-turn injection into a synchrotron. For the first stage of acceleration, it was proposed to use the $100 \mathrm{Mev} / \mathrm{u}$ linac of the EURISOL facility. Further acceleration was to be done with a new rapid cycling synchrotron (RCS), the PS and finally the SPS. A new injection and stacking method was proposed to keep the duty factor of the decay ring low. The method makes use of a dispersion orbit in the decay ring to avoid the injection elements interfering with the circulating beam. Bunch rotation brings the fresh bunches to the central orbit and asymmetric bunch merging to take the newly injected ions into the centre of the circulating bunch [6]. The maximum gamma of 150 that can be reached for fully stripped $6 \mathrm{He}$ ions in the SPS, was initially chosen for the coasting beam in the decay ring but later revised to lower values taking physics reach considerations into account. The main bottlenecks in the scenario chosen for the first study were shown to be the tune shift at PS and SPS injection and the activation of the PS ring. 


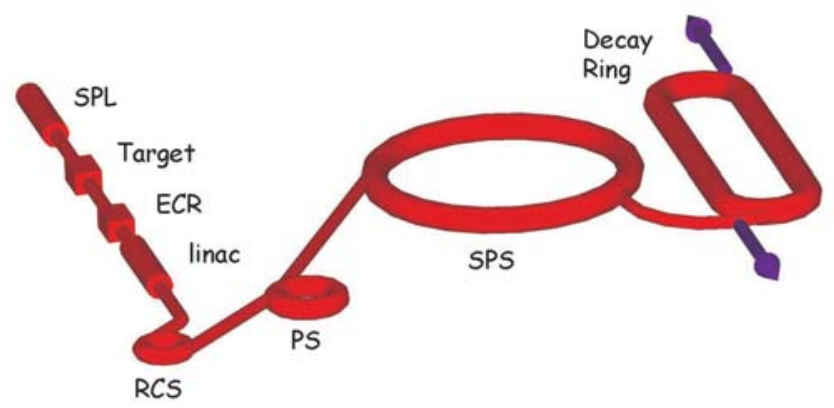

Figure 1: CERN conceptual design

\section{Electron capture decay and beta-beams}

\subsection{Introduction}

The idea to make use of electron-capture decaying nuclei for a beta-beam has recently been discussed [7]. The option of a monochromatic neutrino beam from atomic electron capture in ${ }^{150} \mathrm{Dy}$ has especially received some attention both [6] in its physics reach and in machine feasibility. This nucleus has an unsually short half-life for an electron capture decaying isotope. This is due to super allowed spin-isospin transitions to a giant Gamow-Teller resonance which is kinematically accessible [8]. The short half-life makes it feasible to generate a sufficient neutrino rate from a reasonable amount of stored nuclei (see section 4). In a recent work [9] the possibility to accelerate a fully stripped electron capture decaying isotope has been studied; the isotopes are in this study recombined with an electron beam in the decay ring and would subsequently decay by electron capture. To avoid this complication it is proposed here to accelerate and store partly charged ions.

\subsection{Acceleration and storage of partly charged ions}

The acceleration of partly charged ions is being intensely studied at e.g. GSI for the future FAIR facility [10] and at BNL for the RHIC program. Experience from the Experimental Storage Ring (ESR) at GSI [14] suggests that it it is possible to keep recombination and stripping losses low. At high energy, the stripping cross section will dominate and a very first calculation for the decay ring $[11,13]$ yields less than $5 \%$ of stripping losses per minute. However, the loss rate will strongly depend on the vacuum conditions and the Proton Synchrotron (PS) in the present CERN injector complex is not designed for ultra high vacuum operation. In addition, it has recently been shown [15] that the decay losses will generate a major deterioration of the vacuum conditions in both the PS and SPS towards the end of the acceleration cycle, something which would be unacceptable for the acceleration of a partly charged ion beam. 
The half-life of the partly charged electron capture decaying nuclei with only $\mathrm{n} s$ electrons left will scale as $1 / n^{2}[16,17]$ e.g. a Dy atom with only one $1 s$ electron left would still yield more than $40 \%$ of the yield of a neutral Dy atom.

For the purpose of calculating the neutrino rate it is useful to define an equivalent vacuum half-life and the work done so far indicates that it is reasonable to assume a vacuum half-life of 1 minute during acceleration and 3 minutes in the decay ring. The annual rate of neutrinos can be calculated using the formula given in [18] which in the presence of important vacuum losses must be modified:

$$
R=\frac{I_{\text {inf }} f}{T_{\text {rep }}} \times \frac{\lambda_{\text {ec }} / \gamma_{\text {top }}}{\lambda_{\text {ec }} / \gamma_{\text {top }}+\lambda_{\text {vac }}} \times\left(1-e^{-m T_{\text {rep }}\left(\lambda_{\text {ec }} / \gamma_{\text {top }}+\lambda_{\text {vac }}\right)}\right) \times T_{\text {run }}
$$

where the $m$ is the number of merges that can be done in the decay ring without major losses from the merging process itself, $T_{r e p}$ is the repetition period for the fills in the decay ring, $\gamma_{o p}$ the gamma factor of the decay ring, $\lambda_{e c}$ is the electron capture decay constant at rest, $\lambda_{v a c}$ is the equivalent vacuum decay constant, $I_{\text {in }}$ the total number of ions injected into the decay ring for each fill, $f$ the fraction of the decay ring length for the straight section generating the neutrino beam and $T_{\text {run }}$ the length in seconds of the run (normally taken as $10^{7}$ seconds which equals a snowmass year [23]).

Using the parameters for the second version of the EURISOL beta-beam baseline design [21] and using an accumulator ring at low energy to boost the intensity [19] indicates that with an isotope having an electron capture half-life of 1 minute, a source rate of $10^{13}$ ions per second, a rate of $10^{18}$ neutrinos per snowmass year [23] along one of the straight sections could be achieved. However, it should be noted that the space charge limit of the present CERN injectors is surpassed for such a beam. Furthermore, the electron capture half-life of the best candidate of the newly discovered fast decaying rare-earth isotopes $\left({ }^{148} \mathrm{Dy}\right)$ is 3.1 minutes. The production of this isotope would even at the future EURISOL facility [4] be at least a factor ten below the required $10^{13}$ ions per second.

\subsection{Long half-lives and beta-beams}

The long half-life of most isotopes decaying by electron capture was long thought to make a monochromatic beta-beam impossible. The reason is simply that the flux required would demand a very large number of ions to be stored in the decay ring. At high intensities the beam will be difficult to control due to intra beam scattering, space charge detuning and important vacuum losses. The problems will be further exacerbated by the requirement to keep the duty factor low at the Lorenz gamma values achievable at e.g. the beta-beam facility studied within the EURISOL design study. In table 1 four isotopes discussed in [7] are compared to ${ }^{18} \mathrm{Ne}$ considering the the total number of charges in each of the machines in the EURISOL beta-beam facility and the peak current in the decay ring.

\section{Conclusions}

A mono-chromatic beta beam for the study of neutrino oscillations is probably not an option using any known electron capture decaying nucleus due to too long half-lives and insufficient production rates at the next generation of radioactive beam facilities. The recently discovered nuclei in the rare-earth region come close to fulfilling the requirement of half-lives below a minute. A 
Table 1: The number of charges in each step of the beta-beam facility for a nominal rate of $10^{18}$ neutrinos per snowmass year $\left(10^{7}\right)$ [23] along one straight section of the decay ring. The production rate has been set to $10^{13}$ ions per second which is a factor of 10 above the present estimates for the EURISOL facility. The number of bunches in the decay ring are set to 100 giving a duty factor of $1 \times 10^{-2}$ at $\gamma=100$. For comparison, the nominal values for the ${ }^{18} \mathrm{Ne}$ beam at the nominal flux of $2.1 \times 10^{18}$ neutrinos, $\gamma=100$ and a duty factor of $2 \times 10^{-3}$ are also given. The total number of charges per acceleration cycle for the nominal ${ }^{18} \mathrm{Ne}$ beam is already close to the known limitations of the existing CERN accelerators which are proposed to be re-used for the EURISOL beta-beam facility.

\begin{tabular}{llllll}
\hline Acccelerator & RCS & PS & SPS & DR & DR Peak Current \\
Isotope & {$\left[10^{12} \mathrm{C}\right]$} & {$\left[10^{13} \mathrm{C}\right]$} & {$\left[10^{13} \mathrm{C}\right]$} & {$\left[10^{14} \mathrm{C}\right]$} & {$[\mathrm{kA}]$} \\
\hline${ }^{148} \mathrm{Dy}$ & 120 & 102 & 828 & 87.6 & 3.74 \\
${ }^{150} \mathrm{Dy}$ & 139 & 117 & 948 & 97.8 & 4.18 \\
${ }^{150} \mathrm{Ho}$ & 86.1 & 74.0 & 602 & 68.7 & 2.93 \\
${ }^{152} \mathrm{Tm}$ & 28.3 & 23.2 & 162 & 27.5 & 1.17 \\
\hline${ }^{18} \mathrm{Ne}$ & 2.71 & 4.35 & 4.29 & 7.47 & 1.60 \\
\hline
\end{tabular}

new effort is on its way to re-visit this region of the nuclear chart and measure the electron capture properties of all possible candidates. The best isotope would have a half life of less than a minute with an electron capture decay pre-dominantly feeding one single level in the daughter nucleus. The latter requirement is necessary to avoid several energy peaks in the resulting neutrino energy spectrum. We strongly encourage the search for such an isotope. A monochromatic beta-beam facility could largely be based on the EURISOL beta-beam facility and would only require modifications to the source, the bunching and possible, the vacuum system. Most importantly it would make use of the same decay ring and the same detectors and could consequently represent a future upgrade of a classical beta-beam facility. It is important to determine the full physics reach for oscillation physics with a monchromatic beam to establish if this option should be kept open in future machine studies. The possible use of a low energy monochromatic beta-beam should also be explored.

\section{Acknowledgements}

Many thanks to Michael Benedikt, Fritz Bosch, Adrian Fabich, Steve Hancock, Hsiao-Chaun (Dick) Hseuh, Lukas Lindroos, Thomas Stoehlker and to the collegaues in the EURISOL beta-beam task for helpful discussions and many useful comments.

\section{References}

[1] P.Zucchelli, A novel concept for a neutrino factory: the beta-beam, Phys. Let. B, 532 (2002) 166-172

[2] B.Autin, M. Benedikt, M. Grieser, S. Hancock, H. Haseroth, A. Jansson, U. Köster, M. Lindroos, S. Russenschuck and F. Wenander, The acceleration and storage of radioactive ions for a neutrino factory, CERN/PS 2002-078 (OP), Nufact Note 121, Proceedings of Nufact 02, London, UK, 2002, J. Phys. G: Nucl. Part. Phys. 29 (2003) 1785-1795 
[3] M. Lindroos and T. Nilsson, The acceleration and storage of radioactive ions for a beta-beam facility, Proceedings of RNB6, Argonne, USA, 2003, Nucl. Phys. A 746(2004)316c-322c

[4] http://eurisol.org

[5] H.R. Ravn and B.W. Allardyce, "On-Line Mass Separators”, in Treatise on Heavy-Ion Science, Edt. D. A. Bromley, Plenum Press, New York, 1989, ISBN 0-306-42949-7

[6] M. Benedikt, S. Hancock, "A novel scheme for injection and stacking of radioactive ions at high energy”, NIM A 550(2005)1

[7] J. Bernabeu, J. Burguet-Castell, C. Espinoza and M. Lindroos, "Monochromatic neutrino beams", JHEP12(2005)014

[8] E. Nacher, Beta decay studies in the $N \sim Z$ and the rare-earth regions using Total Absorption Spectroscopy techniques, Ph. D. Thesis, Univ. Valencia (2004).

[9] J. Sato, "Monoenergetic Neutrino Beam for Long-Baseline Experiments", Phys. Rev. Lett. 95(2005)131804

[10] R. D. DuBois, A. C. F. Santos, Th. Stohlker, F. Bosch, A. Bräuning-Demian, A. Gumberidze, S. Hagmann, C. Kozhuharov, R. Mann, A. OrŽiæ Muthig, U. Spillmann, S. Tachenov, W. Bart, L. Dahl, B. Franzke, J. Glatz, L. Gröning, S. Richter, D. Wilms, K. Ullmann and O. Jagutzki, Phys.Rev. A 70(2004)032712

[11] A. S. Schlachter, J. W. Stearns, W. G. Graham, K. H. Berkner, R. V. Pyle, and J. A. Tanis, "Electron capture for fast highly charged ions in gas targets: An empirical scaling rule", Phys. Rev. A 27(1983)3372

[12] H-.D. Betz, "Charge States and Charge-Changing Cross Sections of Fast Heavy Ions Penetrating Through Gaseous and Solid Media”, Rev. Mod. Phys. 44(1972)465

[13] H. Hseuh, BNL, USA, 2005, private communication

[14] B. Franzke, "Vacuum Requirements for Heavy Ion Synchrotrons", IEEE Transactions on Nuclear Science, Vol. NS-28, No. 3, June 1981

[15] A. Fabich and M. Benedikt, Decay losses along the accelerator chain of the Beta-beam, EURISOL DS/TASK12/TN-05-05

[16] F. Bosch, GSI, Germany, 2005, private communication

[17] W. Bambynek, H. Behrens, M. H. Chen, B. Crasemann, M. L. Fitzpatrick, K. W. D. Ledingham, H. Genz, M. Mutterer and R. L. Intemann, "Orbital electron capture by the nucleus", Rev. Mod. Phys. 49(1977)77

[18] M. Benedikt, A. Fabich, S. Hancock and M. Lindroos, in the proceedings of NUFACT05, Frascati, Italy, 2005

[19] A. Källberg and M. Lindroos, "Accumulation in a ring at low energy for the beta-beam", EURISOL DS/TASK12/TN-05-04, http://eurisol.org

[20] M. Benedikt, S. Hancock, M. Lindroos, "Parameter and Intensity Values, Version 1, April 2005", EURISOL DS/TASK12/TN-05-01, http://eurisol.org

[21] M. Benedikt, A. Fabich, S. Hancock, M. Lindroos, "Parameter and Intensity Values, Version 2, July 2005", DS/TASK12/TN-05-03, http://eurisol.org 
[22] “The EURISOL report”, Edt. J. Cornell, GANIL, Caen, 2003, European commission contract No. HPRI-CT-1999-500001

[23] http://snowmass2001.org/ 